\title{
Exponentially Small Cosmological Constant in String Theory
}

\author{
Mehmet Demirtas@, Manki Kim๑, Liam McAllister®, Jakob Moritz®, and Andres Rios-Tascon \\ Department of Physics, Cornell University, Ithaca, New York 14853, USA
}

(Received 25 July 2021; accepted 23 November 2021; published 4 January 2022)

\begin{abstract}
We construct a supersymmetric vacuum of string theory in which the magnitude of the vacuum energy is $<10^{-123}$ in Planck units. The mechanism that we introduce generates a well-controlled exponential hierarchy from an appropriate choice of the quantized parameters in the superpotential. Our approach provides a new angle on the cosmological constant problem.
\end{abstract}

DOI: 10.1103/PhysRevLett.128.011602

Introduction.-Explaining the smallness of the observed dark energy density $[1,2]$,

$$
\rho_{\mathrm{obs}} \approx 10^{-123} M_{\mathrm{pl}}^{4},
$$

is a profound challenge [3-5]. The idea that anthropic selection in a landscape could account for the smallness of $\rho_{\text {obs }}$ [6] has been influential and, in broad strokes, appears to align with known and suspected properties of string theory [7]. Toy landscapes of flux configurations in compactifications of string theory [8-10] admit a vast number $\mathcal{N}_{\text {vac }}$ of solutions, and the values that the classical vacuum energy $\rho_{\text {class }}$ takes in such ensembles can be finely spaced, with differences $\Delta \rho_{\text {class }} \sim M_{\text {pl }}^{4} / \mathcal{N}_{\text {vac }} \ll \rho_{\text {obs }}$.

One natural objection is that the true explanation might be a richer one, involving dynamics or a different sort of mechanism, and we might fail to seek it by too-readily accepting an anthropic approach. We will do nothing to overcome this concern, remarking only that, in the absence of any framework for a dynamical explanation, perhaps the best one can do is to sharpen the landscape argument.

A more practical complaint is that exploring a landscape of vacua of string theory and finding solutions therein, in which the actual value $\rho_{\text {vac }}$ of the vacuum energy obeys $\rho_{\mathrm{vac}} \approx \rho_{\text {obs }}$, appears out of reach. The mechanism proposed by Bousso and Polchinski [7] relies on the high dimensionality of the space of fluxes, and so one faces a search for special, exponentially rare solutions in a highdimensional energy landscape. At least in its full generality, this problem's computational complexity makes it inaccessible by a direct assault [11-13]. Even worse, given a candidate de Sitter vacuum in string theory, one can at best imagine computing $\rho_{\text {vac }}$ order by order and

Published by the American Physical Society under the terms of the Creative Commons Attribution 4.0 International license. Further distribution of this work must maintain attribution to the author(s) and the published article's title, journal citation, and DOI. Funded by SCOAP. nonperturbatively: in the string loop expansion, in the $\alpha^{\prime}$ expansion, and perhaps in other approximations at the same time. Achieving in this way a precision of order $\rho_{\text {obs }}$ does not seem possible to us. The difficulty of exhibiting de Sitter vacua of string theory with $\rho_{\text {vac }} \approx \rho_{\text {obs }}$ thus presents an obstruction to bringing the landscape argument for the cosmological constant problem into sharp focus in quantum gravity.

An essential part of the problem is achieving scale separation in cosmological solutions of quantum gravity: can one find isolated solutions of string theory in which the Kaluza-Klein radius $R_{\mathrm{KK}}$ of the internal space is not extremely large in units of the Planck length $\ell_{p}$, and yet the noncompact spacetime has a cosmologically large radius of curvature $R_{\text {cosm }} \sim 10^{60} \ell_{p}$ ? In a theory with unbounded continuous parameters, this question might not arise. However, the known classes of realistic solutions of string theory come in large but finite families (see, however, [14]), and their low-energy parameters are ultimately determined by inherently quantized parameters of the string vacuum, such as the topological data of a Calabi-Yau flux compactification. Whether or not $R_{\text {cosm }} \gtrsim 10^{60} \ell_{p}$ can occur in a particular setting evidently depends on the ranges of values that these quantized parameters can take and on how $\rho_{\text {vac }}$ depends on these parameters.

In this Letter, we exhibit a solution of type IIB string theory with $\left|\rho_{\mathrm{vac}}\right| \approx 10^{-144} M_{\mathrm{pl}}^{4}$, in which the internal space-an orientifold of a Calabi-Yau threefold-has radius $R_{\mathrm{KK}} \approx 10^{4} \ell_{p}$. This vacuum is an example of extreme scale separation, but in four-dimensional anti-de Sitter space $\left(\mathrm{AdS}_{4}\right)$, not $\mathrm{dS}_{4}$ : the solution preserves $\mathcal{N}=1$ supersymmetry, and the cosmological constant is negative.

Although this compactification cannot describe our Universe, it provides an intriguing angle on the cosmological constant problem. To stumble upon a solution with vacuum energy $\varepsilon M_{\mathrm{pl}}^{4}$ in a complex landscape of vacua in which the characteristic scale is $M_{\mathrm{pl}}$, one would naively expect to have to explore $\mathcal{O}(1 / \varepsilon)$ distinct vacua. The actual distribution of cosmological constants could play a role, of 
course, but the above expectation should be a reasonable guide unless the set of solutions manifests an exponentially strong concentration around $\rho=0$-which is to say, unless the landscape furnishes a bona fide statistical solution to the cosmological constant problem, a possibility that we shall discount in this Letter. In particular, to find vacuum energy $\varepsilon M_{\mathrm{pl}}^{4}$ in a high-dimensional landscape of flux vacua, one generally has to search through $\mathcal{O}(1 / \varepsilon)$ choices of quantized flux.

We have arrived at $\left|\rho_{\text {vac }}\right| \ll 10^{-123} M_{\mathrm{pl}}^{4}$ without performing such a costly search. Instead, we have exploited structures in the quantized parameters occurring in string compactifications: specifically, 3-form flux quanta in an orientifold of a Calabi-Yau threefold $X$ and the GopakumarVafa invariants $[15,16]$ of curves in the mirror threefold $\tilde{X}$. We have found choices of flux for which the part of the flux superpotential [17] that descends from perturbative contributions to the prepotential in compactification of type IIA string theory on $\tilde{X}$ vanishes exactly, while the nonperturbative terms, which arise from worldsheet instantons on $\tilde{X}$, fall into the form of a racetrack. The competition of two such instanton terms then generates an exponentially small number [18]. When the fluxes and Gopakumar-Vafa invariants are modest integers like 1 or 2 , the resulting hierarchy is similarly modest. But there exist, and we have found, CalabiYau threefolds for which the integers in question are, for example, 2 and 252, and the hierarchy in the vacuum energy is proportional to

$$
\left(\frac{2}{252}\right)^{58} \approx 10^{-122}
$$

In this Letter, we will present one such example.

A Vacuum.-To define a compactification [19] of type IIB string theory on an orientifold of a Calabi-Yau threefold hypersurface $X$ in a toric variety $V$, we consider the reflexive polytope $\Delta[21]$ with vertices given by the columns of

$$
\left(\begin{array}{cccccccc}
1 & -3 & -3 & 0 & 0 & 0 & -5 & -2 \\
0 & -2 & -1 & 0 & 0 & 1 & -3 & -1 \\
0 & 0 & -1 & 0 & 1 & 0 & 0 & 1 \\
0 & 0 & 0 & 1 & 0 & 0 & -1 & -1
\end{array}\right) .
$$

We define toric varieties $\tilde{V}$ and $V$, respectively, in terms of fine regular star triangulations of $\Delta$ and its polar dual $\Delta^{\circ}$. The anticanonical hypersurfaces in $\tilde{V}$ and $V$ are a mirror pair of Calabi-Yau threefolds $\tilde{X}$ and $X$, with $h^{1,1}(X)=h^{2,1}(\tilde{X})=113$, and $h^{2,1}(X)=h^{1,1}(\tilde{X})=5$. We find an O3/O7-orientifold involution of $X$ with 26 O7planes and 48 O3-planes, and obeying $h_{-}^{1,1}=h_{+}^{2,1}=0$. The D3-brane tadpole is 60 .
In this compactification, the 3-form fluxes

$\begin{aligned} \vec{f} & =\left(\begin{array}{llllllllllll}10 & 12 & 8 & 0 & 0 & 4 & 0 & 0 & 2 & 4 & 11 & -8\end{array}\right), \\ \vec{h} & =\left(\begin{array}{llllllllllll}0 & 8 & -15 & 11 & -2 & 13 & 0 & 0 & 0 & 0 & 0 & 0\end{array}\right),\end{aligned}$

which carry D3-brane charge 56, lead to an exponentially small flux superpotential. The dominant instantons along the perturbatively flat valley, as defined in [18], have Gopakumar-Vafa invariants -2 and 252, respectively. The flux superpotential takes the form

$$
W_{\text {flux }}(\tau)=\sqrt{\frac{2^{3}}{\pi^{5}}}\left(-2 e^{2 \pi i \tau \frac{7}{29}}+252 e^{2 \pi i \tau \frac{7}{28}}\right) M_{\mathrm{pl}}^{3},
$$

up to corrections of order $\mathcal{O}\left(e^{2 \pi i \tau(43 / 116)}\right)$. The string coupling is $g_{s} \approx 0.011$, and we find

$W_{0}:=\left\langle\left|W_{\text {flux }}\right|\right\rangle \approx 0.526 \times\left(\frac{2}{252}\right)^{29} M_{\mathrm{pl}}^{3} \approx 6.46 \times 10^{-62} M_{\mathrm{pl}}^{3}$.

Turning now to the Kähler moduli, we find 114 prime toric divisors $D_{I}$ that are rigid and whose uplifts to divisors in Ftheory have trivial intermediate Jacobian. These divisors therefore generate nonvanishing superpotential terms [22], and their Pfaffian prefactors $\mathcal{A}_{D_{I}}$ are pure constants [23]. Thus the total superpotential takes the form

$$
W=W_{0}+\sum_{I} \mathcal{A}_{D_{I}} \exp \left(-\frac{2 \pi}{c_{I}} T_{D_{I}}\right)+\cdots
$$

where $c_{I}=6$ if there is an so(8) stack of seven-branes on $D_{I}$, and $c_{I}=1$ otherwise.

With this superpotential, we find a $\mathcal{N}=1$ supersymmetric $\mathrm{AdS}_{4}$ vacuum at which the volume of the internal space in string units is $\mathcal{V} \approx 945$. At this point in Kähler moduli space there are perturbative and nonperturbative corrections to the Kähler potential $\mathcal{K}$, but these lead to a controllably small multiplicative correction to the vacuum energy [20]. The overall scale of the vacuum energy is dictated by the superpotential, which does not suffer renormalization at any perturbative order [24,25]. Convergence of the series of worldsheet instanton corrections to $\mathcal{K}$ is shown in Fig. 1.

We have therefore found a supersymmetric $\mathrm{AdS}_{4}$ vacuum with vacuum energy

$$
V_{0}=-3 M_{\mathrm{pl}}^{-2} e^{\mathcal{K}}|W|^{2} \approx-1.68 \times 10^{-144} M_{\mathrm{pl}}^{4} \text {. }
$$

This vacuum is very well controlled as a result of the residual supersymmetry and the smallness of the string 

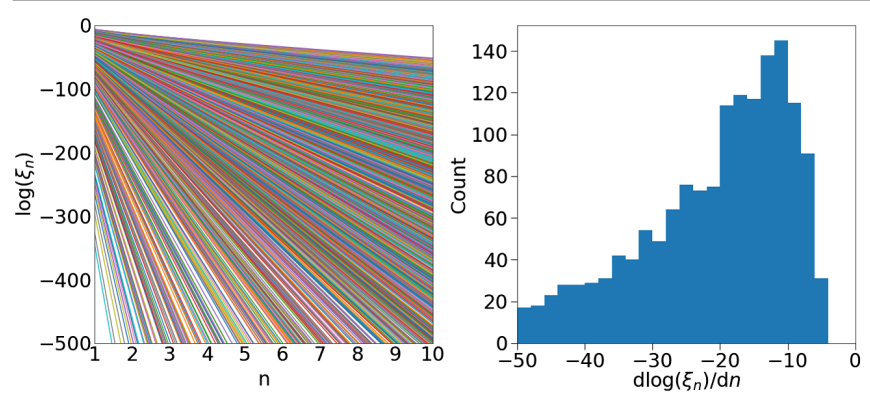

FIG. 1. Left: the logarithm of the size $\xi_{n}$ of the $n$th term in the series of worldsheet instantons resulting from a sample of 1728 rays in $\mathrm{H}_{2}(X)$. Right: histogram of the slopes of the lines in the left panel. The sum evidently converges.

coupling. The Kaluza-Klein radius is $R_{\mathrm{KK}} \approx 10^{4} \ell_{p}$, so the hierarchy of scales is $R_{\text {cosm }} / R_{\mathrm{KK}} \approx 10^{68}$.

We have constructed a number of comparable vacua in compactifications on orientifolds of Calabi-Yau hypersurfaces with $4 \leq h^{2,1} \leq 7$ and $51 \leq h^{1,1} \leq 214$ [20].

Discussion.-The cosmological constant problem demands that we explain how exponentially large universes arise in theories with small fundamental length scales. In this Letter, we have taken a step forward by showing how an exponentially large supersymmetric universe can arise in string theory. Specifically, we exhibited an $\mathrm{AdS}_{4}$ solution of string theory in which the AdS length is approximately $10^{72} \ell_{p}$, which is much larger than the diameter of the observable Universe in which we live. Our construction thus solves a supersymmetric version of the cosmological constant problem and may shine a light toward a solution of the much harder general problem.

A key mechanism underlying our class of constructions is the exact cancellation of all perturbative terms in the superpotential. Specifically, we have arranged the quantized 3 -form fluxes so that all perturbative [26] contributions to the flux superpotential cancel perfectly: this is possible because the superpotential is ultimately determined by integer data. All remaining terms in the scalar potential are proportional to nonperturbative effects. We have made further discrete choices to arrange that these effects balance in a controlled minimum, finding an orientifold of a Calabi-Yau threefold $X$ that supports an array of nonperturbative superpotential terms from Euclidean D3-branes and gaugino condensation, and whose mirror $\tilde{X}$ enjoys a pattern of Gopakumar-Vafa invariants that give rise to a racetrack of worldsheet instantons [18]. These choices lead to stabilization of all the moduli in a supersymmetric vacuum, as foreseen in [27], with a cosmological constant that is exponentially small in Planck units.

The smallness of the vacuum energy in our construction is natural, in the sense that it is determined by a competition among exponentials, once we have ensured the absence of all perturbative terms in the superpotential by making discrete choices of topology and fluxes. Of course, it is very well known that small scales can arise dynamically in supersymmetric theories [28], and in fourdimensional $\mathcal{N}=1$ effective supergravity one can easily write a racetrack superpotential whose minimization yields a supersymmetric $\mathrm{AdS}_{4}$ vacuum with small vacuum energy. But presented with such an effective description, one could ask how finely balanced a racetrack is allowed by ultraviolet completion in quantum gravity. Thus, something is gained by realizing such a construction in string theory, where all the underlying parameters are quantized, and their allowable values can be determined.

The principal result of this Letter is our demonstration, through the explicit example of Sec. II, that exponentially small vacuum energy, on a scale comparable to the observed dark energy density in our Universe, is possible in a compactification of string theory. Our solution is not realistic: the cosmological constant is negative, and we have not addressed the fundamental problem of arranging for small positive vacuum energy after supersymmetry breaking, electroweak symmetry breaking, and the QCD phase transition. However, as we explained in the Introduction, our result is already very surprising from the perspective of prior studies of the statistics of string vacua [8-10] and of the computational complexity of finding small vacuum energy therein [11-13] by choosing appropriate flux quanta [7]. In this sense, we have uncovered a new perspective on the cosmological constant problem: one can speculate that the smallness of the observed vacuum energy in our Universe could be governed by nonperturbative effects in a configuration in which perturbative contributions exactly vanish, and the exactness of the cancellation is ensured by the quantization of fundamental parameters.

Our construction is, at the same time, the first explicit example of a vacuum with a hierarchically small cosmological constant in the celebrated framework proposed by Kachru et al. (KKLT) [27] (for important early works in this direction, see [29-31]). The methods laid out in this Letter allow for construction of ensembles of explicit and well-controlled KKLT vacua [20] and so pave the way for direct exploration of a corner of the landscape of string theory that has been the subject of intense investigation without a corresponding wealth of examples.

Exploring solutions of the sort we have presented here may lead to an improved understanding of the cosmological constant problem in quantum gravity.

We thank Yuval Grossman for inspiring discussions, and we are indebted to Naomi Gendler, Ben Heidenreich, Tom Rudelius, and Mike Stillman for collaborations on related topics. The research of M. D., M. K., L. M., and A. R.-T. was supported in part by NSF Grant No. PHY-1719877, and that of L. M. and J. M. was supported in part by the Simons Foundation Origins of the Universe Initiative. 
[1] A. G. Riess et al. (Supernova Search Team Collaboration), Observational evidence from supernovae for an accelerating universe and a cosmological constant, Astron. J. 116, 1009 (1998).

[2] S. Perlmutter et al. (Supernova Cosmology Project Collaboration), Measurements of $\Omega$ and $\Lambda$ from 42 high redshift supernovae, Astrophys. J. 517, 565 (1999).

[3] S. Weinberg, The cosmological constant problem, Rev. Mod. Phys. 61, 1 (1989).

[4] J. Polchinski, The cosmological constant and the string landscape, in Proceedings of the 23rd Solvay Conference in Physics: The Quantum Structure of Space and Time (2006).

[5] R. Bousso, TASI lectures on the cosmological constant, Gen. Relativ. Gravit. 40, 607 (2008).

[6] S. Weinberg, Anthropic Bound on the Cosmological Constant, Phys. Rev. Lett. 59, 2607 (1987).

[7] R. Bousso and J. Polchinski, Quantization of four form fluxes and dynamical neutralization of the cosmological constant, J. High Energy Phys. 06 (2000) 006.

[8] S. Ashok and M. R. Douglas, Counting flux vacua, J. High Energy Phys. 01 (2004) 060.

[9] F. Denef and M. R. Douglas, Distributions of flux vacua, J. High Energy Phys. 05 (2004) 072.

[10] M. R. Douglas and S. Kachru, Flux compactification, Rev. Mod. Phys. 79, 733 (2007).

[11] F. Denef and M. R. Douglas, Computational complexity of the landscape. I., Ann. Phys. (Amsterdam) 322, 1096 (2007).

[12] F. Denef, M. R. Douglas, B. Greene, and C. Zukowski, Computational complexity of the landscape IICosmological considerations, Ann. Phys. (Amsterdam) 392, 93 (2018).

[13] J. Halverson and F. Ruehle, Computational complexity of vacua and near-vacua in field and string theory, Phys. Rev. D 99, 046015 (2019).

[14] O. DeWolfe, A. Giryavets, S. Kachru, and W. Taylor, Type IIA moduli stabilization, J. High Energy Phys. 07 (2005) 066.

[15] R. Gopakumar and C. Vafa, M theory and topological strings. 1, arXiv:hep-th/9809187.

[16] R. Gopakumar and C. Vafa, M theory and topological strings. 2, arXiv:hep-th/9812127.
[17] S. Gukov, C. Vafa, and E. Witten, CFT's from Calabi-Yau four folds, Nucl. Phys. B584, 69 (2000); B608, 477(E) (2001).

[18] M. Demirtas, M. Kim, L. McAllister, and J. Moritz, Vacua with Small Flux Superpotential, Phys. Rev. Lett. 124, 211603 (2020).

[19] Extensive discussion of this and other examples can be found in a companion paper [20]: in particular, the full data of the construction are available in the Supplemental Material associated with [20].

[20] M. Demirtas, M. Kim, L. McAllister, J. Moritz, and A. RiosTascon, Small cosmological constants in string theory, arXiv:2107.09064 [J. High Energy. Phys. (to be published)].

[21] M. Kreuzer and H. Skarke, Complete classification of reflexive polyhedra in four-dimensions, Adv. Theor. Math. Phys. 4, 1209 (2000).

[22] E. Witten, Nonperturbative superpotentials in string theory, Nucl. Phys. B474, 343 (1996).

[23] E. Witten, Five-brane effective action in M theory, J. Geom. Phys. 22, 103 (1997).

[24] M. Dine and N. Seiberg, Nonrenormalization Theorems in Superstring Theory, Phys. Rev. Lett. 57, 2625 (1986).

[25] C. P. Burgess, C. Escoda, and F. Quevedo, Nonrenormalization of flux superpotentials in string theory, J. High Energy Phys. 06 (2006) 044.

[26] The Gukov-Vafa-Witten flux superpotential [17] in a type IIB compactification on a Calabi-Yau threefold $X$ is purely classical, but in an equivalent mirror dual type IIA formulation contains both perturbative and nonperturbative terms.

[27] S. Kachru, R. Kallosh, A. D. Linde, and S. P. Trivedi, De Sitter vacua in string theory, Phys. Rev. D 68, 046005 (2003).

[28] E. Witten, Dynamical breaking of supersymmetry, Nucl. Phys. B188, 513 (1981).

[29] F. Denef, M. R. Douglas, and B. Florea, Building a better racetrack, J. High Energy Phys. 06 (2004) 034.

[30] F. Denef, M. R. Douglas, B. Florea, A. Grassi, and S. Kachru, Fixing all moduli in a simple f-theory compactification, Adv. Theor. Math. Phys. 9, 861 (2005).

[31] D. Lust, S. Reffert, E. Scheidegger, W. Schulgin, and S. Stieberger, Moduli stabilization in type IIB orientifolds (II), Nucl. Phys. B766, 178 (2007). 